\title{
MODELO DE PROGRAMAÇÃO QUADRÁTICA PARA ANÁLISE DA MOVIMENTAÇÃO LOGÍSTICA E COMERCIALIZAÇÃO DA SOJA BRASILEIRA ${ }^{1}$
}

\author{
ANDRÉA L. R. O. OJIMA ${ }^{2}$, AKEBO YAMAKAMI ${ }^{3}$
}

\begin{abstract}
RESUMO: A proposta deste trabalho é analisar a distribuição logística da soja brasileira aplicando-se modelo de equilíbrio espacial de programação quadrática. O sistema de transporte da soja é um ponto importante na cadeia produtiva, pois a maior parte dos custos dessa commodity é dada pelos custos de transporte. Nesse sentido, a otimização desse segmento é essencial para aumentar a competitividade da soja brasileira no mercado internacional. O complexo soja brasileiro tem aumentado a sua participação nas exportações dos produtos agrícolas nos últimos dez anos, mas os investimentos de outros países faz com que as exportações brasileiras não fiquem focadas apenas no crescimento da produção, mas, sim, na eficiência de toda a cadeia produtiva. Dessa maneira, com a aplicação de um modelo de equilíbrio espacial de programação quadrática, pode-se projetar novos cenários alterando-se os custos de transporte dos diferentes modais, conduzindo políticas para novos investimentos no setor de transportes.
\end{abstract}

PALAVRAS-CHAVE: transporte de soja, logística, programação matemática.

\section{QUADRATIC PROGRAMMING MODEL IN THE ANALYSIS OF THE LOGISTICAL MOVEMENT AND MARKETING OF BRAZILIAN SOYBEAN}

\begin{abstract}
The purpose of this work was to analyze the logistical distribution of Brazilian soybean by applying a quadratic programming to a spatial equilibrium model. The soybean transportation system is an important part of the soybean complex in Brazil, since the major part of the costs of this commodity derives from the transportation costs. Therefore, the optimization of this part of the process is essential to a better competitiveness of the Brazilian soybean in the international market. The Brazilian soybean complex have been increasing its agricultural share in the total of the exportation value in the last ten years, but due to other countries' investments the Brazilian exportations cannot be only focused on increasing its production but it still have to be more efficient. This way, a model was reached which can project new frames by switching the transportation costs and conduce the policy makers to new investments in the sector.
\end{abstract}

KEYWORDS: soybean transportation, logistics, mathematical programming.

\section{INTRODUÇÃO}

A soja chegou ao Brasil com os primeiros imigrantes japoneses, em 1908, mas foi introduzida, oficialmente, no Rio Grande do Sul, em 1914. Entretanto, a sua expansão deu-se efetivamente a partir dos anos de 1970, com o interesse crescente da indústria de óleo e a demanda do mercado internacional. A criação de novas cultivares pelos melhoristas levou a soja para diversas regiões brasileiras, dando estabilidade às áreas de fronteira agrícola do País (OJIMA, 2004).

O complexo soja, isto é, grão-farelo-óleo, constitui-se numa das mais importantes commodities nacionais, sendo responsável, na última safra, pela captação de divisas no mercado internacional da ordem de US\$ 10 bilhões, de acordo com dados do Ministério do Desenvolvimento, Indústria e Comércio Exterior (MDIC).

\footnotetext{
${ }^{1}$ Extraído da Dissertação de Mestrado do primeiro autor. Apresentado no XXXIII Congresso Brasileiro de Engenharia Agrícola.

${ }^{2}$ Eng $^{\mathrm{a}}$ Agrônoma, Mestre em Engenharia Elétrica, Pesquisadora, Instituto de Economia Agrícola (IEA), São Paulo - SP, Fone: (0XX11) 5067.0481, andrea@iea.sp.gov.br.

${ }^{3}$ Professor Titular, Faculdade de Engenharia Elétrica e de Computação, UNICAMP, Campinas - SP.

Recebido pelo Conselho Editorial em: 5-7-2004
}

Aprovado pelo Conselho Editorial em: 25-4-2006 
A soja movimenta grande montante de recursos e divisas, mas por ser um produto de baixo valor agregado, é necessário que haja otimização da produção, estocagem e transporte. Assim, técnicas de modelagem para a otimização dessa cadeia estão sendo utilizadas para tentar reduzir os custos do transporte, tornando-a mais atraente e lucrativa para os produtores e investidores.

A melhoria nos sistemas de transporte corresponde ao maior diferencial nos preços, dado que esse estágio absorve cerca de $30 \%$ dos gastos do complexo soja no Brasil (Tabela 1).

TABELA 1. Composição dos custos da soja produzida nos Estados Unidos (Illinois) e Brasil (Mato Grosso) com destino ao Porto de Rotterdam na Holanda (US\$ t ${ }^{-1}$ ), em 2003.

\begin{tabular}{l|c|c}
\hline & Estados Unidos (Illinois) & Mato Grosso (Sorriso) \\
\hline Custo de Produção (A) & 203,5 & 174,0 \\
\hline Frete ao porto & 26,0 & 61,9 \\
Tarifa portuária & 3,0 & 7,0 \\
Frete marítimo até Rotterdam & 21,4 & 30,6 \\
\hline Custo de transporte (B) & 50,4 & 99,5 \\
\hline Prêmio & $(13,0)$ & 80 \\
\hline Custo A + B & 240,9 & 353,5 \\
\hline \% do custo de transporte & $20,9 \%$ & $28,1 \%$ \\
\hline
\end{tabular}

Fonte: adaptado de TAVARES (2004); BRASIL FERROVIAS (2003); LIMA et al. (2005).

O cenário do setor dos transportes pode ser caracterizado pela predominância da movimentação de produtos de baixo valor agregado percorrendo longas distâncias. Desse modo, seria necessário favorecer arranjos logísticos que contemplassem o transporte hidroviário e ferroviário, indicados para esse tipo de perfil.

No período compreendido entre 1970 e 2004, a modalidade de transporte rodoviário havia absorvido mais da metade dos transportes de cargas no Brasil, sendo em 2004 responsável por $61,1 \%$ contra $20,7 \%$ do transporte ferroviário, $13,6 \%$ do transporte aquaviário e 4,6\% de outros (duto e aéreo) (CONFEDERAÇÃO NACIONAL DO TRANSPORTE, 2005). Conforme CAIXETA FILHO (1996), "essa predominância do modo rodoviário pode ser explicada pelas dificuldades que outras categorias de transporte enfrentam para atender eficientemente aos aumentos de demanda em áreas mais afastadas do País, as quais não são servidas por ferrovias ou hidrovias".

O aproveitamento do potencial de expansão da produção de grãos depende do estabelecimento de um sistema eficiente de transporte. LíCIO (1995) ressalta a relevância da viabilização e da integração dos corredores de transportes multimodais (rodovia, ferrovia, hidrovia) para aumentar a competitividade dos produtos, integrando as áreas de produção, os centros consumidores e o mercado internacional. Os modelos de equilíbrio espacial, que utilizam ferramentas de programação quadrática, têm sido usados pela economia agrícola para simular o impacto de novas medidas sobre o setor, além de nortear as mudanças nas políticas de transportes.

No presente trabalho, teve-se como objetivo a aplicação de um modelo de equilíbrio espacial utilizando técnicas de programação quadrática para a movimentação da soja brasileira. A solução sugerida pelo modelo determina o fluxo de soja-grão das regiões de oferta para as regiões de demanda doméstica, portos e demanda internacional, associados com as principais mudanças com que novos projetos viários possam vir a ocasionar.

\section{MATERIAL E MÉTODOS}

Produção e/ou consumo usualmente ocorrem em regiões separadas espacialmente, cada uma delas possuindo relação de oferta e demanda. Se os preços entre as regiões forem dados em maior grau pelos custos de transporte inter-regionais, a comercialização ocorrerá e será direcionada por tais custos. Modelando essa situação, colocam-se pelo menos algumas questões a serem 
respondidas, dentre as quais, quem irá produzir e consumir, em que quantidades e em que níveis a comercialização se dará.

As condições de equilíbrio espacial para commodities com custos de transporte ligados às regiões de exportação e importação podem ser vistas na Figura 1, em que $\mathrm{P}$ corresponde ao preço e $\mathrm{Q}$ à quantidade. A quantidade da commodity negociada é igual ao excesso de oferta (ef) na região de exportação, enquanto a quantidade importada é igual ao excesso de demanda (gh) no preço de equilíbrio, assumindo que o custo de transporte é igual a zero entre as duas regiões. Com a existência de tarifas e custos de transporte medidos pela distância vertical entre as curvas de importação demandada e a exportação ofertada (ab), a diferença do preço entre as regiões de importação e exportação é igual às tarifas e custos de transporte. Esses custos e tarifas são compartilhados pelas regiões de exportação e importação, de acordo com as respectivas elasticidades de cada região.

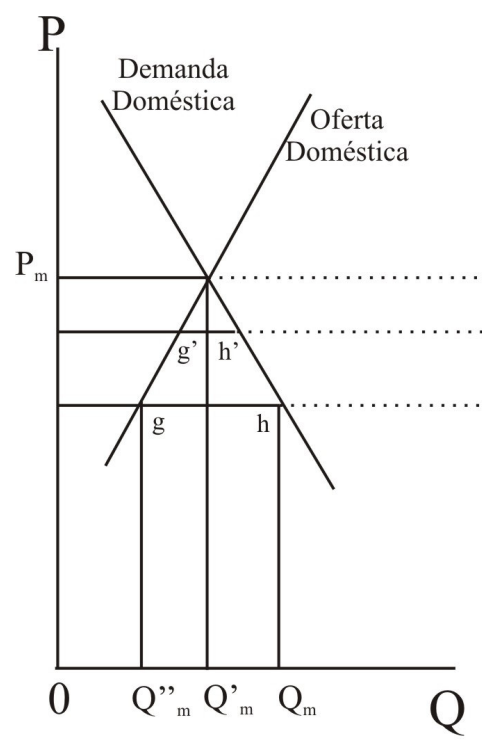

Região Importadora

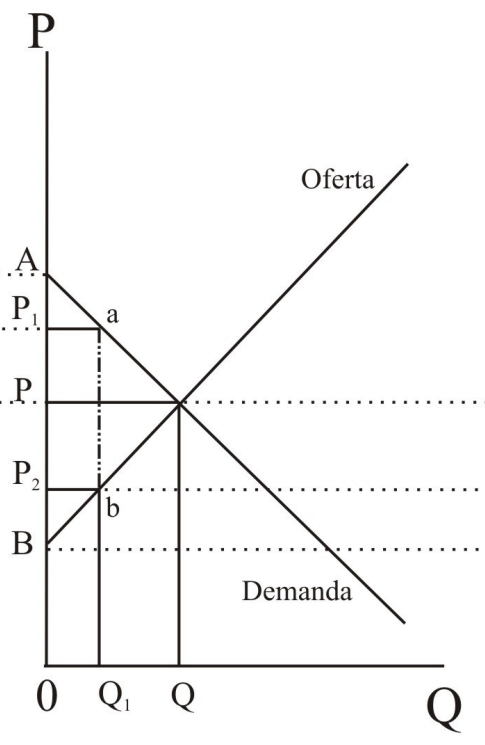

Mercado Internacional

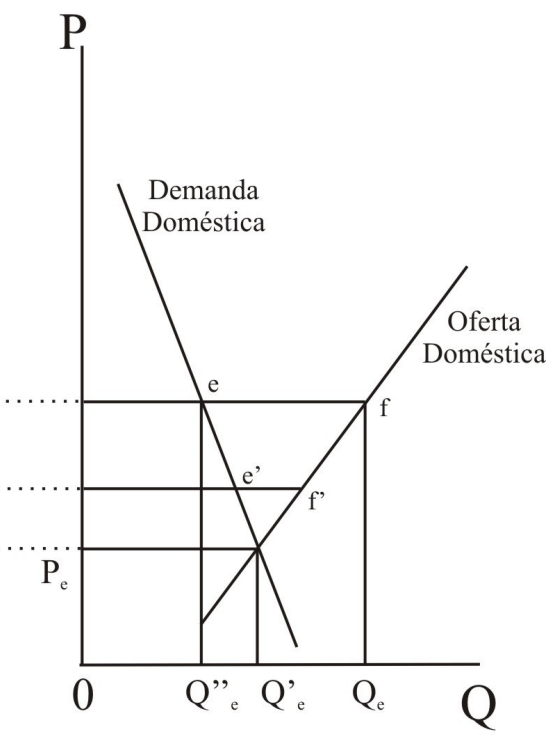

Região Exportadora

FIGURA 1. Equilíbrio internacional de comercialização entre duas regiões. Fonte: FELLIN (1993).

Na Figura 1, as tarifas e custos de transporte (ab) aumentam o preço na região importadora de $\mathrm{P}$ para $\mathrm{P} 1$, assim o aumento do preço pago pela região importadora resulta em decréscimo na quantidade comercializada de Q para Q1. A proporção do diferencial do preço pago pelos produtores das regiões exportadoras (P2) e a incorporação para os consumidores na importação (P1) podem ser calculadas com a função das elasticidades de oferta e demanda.

A estrutura teórica desse modelo pode ser expandida, incluindo regiões multi-exportadoras e importadoras, transporte multimodal e multicommodity. Os problemas de equilíbrio espacial são expressos matematicamente, por meio da maximização das áreas sob as curvas de demanda menos as áreas sob as curvas de oferta e menos os custos de transporte. Por exemplo, $i$ regiões de comércio substituem duas regiões descritas na Figura 1, fazendo com que a demanda na região $i$ seja dada por:

$$
\mathrm{p}_{\mathrm{i}}=\mathrm{d}_{\mathrm{i}}\left(\mathrm{y}_{\mathrm{i}}\right)
$$

em que,

$\mathrm{p}_{\mathrm{i}}$ - preço de demanda na região $i$, e

$\mathrm{y}_{\mathrm{i}}$ - quantidade de demanda com preço $\mathrm{p}_{\mathrm{i}}$ na região $i$.

A função de oferta para a região é definida por:

$$
\mathrm{p}^{\mathrm{i}}=\mathrm{s}_{\mathrm{i}}\left(\mathrm{z}_{\mathrm{i}}\right)
$$

em que, 
$\mathrm{p}^{\mathrm{i}}$ - preço de oferta na região $i, \mathrm{e}$

$\mathrm{Z}_{\mathrm{i}}$ - quantidade ofertada com preço $\mathrm{p}^{\mathrm{i}}$ na região $i$.

Assumindo que a inclinação da curva de demanda é descendente e que a inclinação da curva de oferta é ascendente, tem-se que:

$$
\partial\left(\mathrm{d}_{\mathrm{i}}\left(\mathrm{y}_{\mathrm{i}}\right)\right) / \mathrm{y}_{\mathrm{i}}<0 \quad \text { e } \quad \partial\left(\mathrm{s}_{\mathrm{i}}\left(\mathrm{z}_{\mathrm{i}}\right)\right) / \mathrm{z}_{\mathrm{i}} \geq 0
$$

A função-lucro é definida como a área abaixo da curva da demanda menos a área abaixo da curva de oferta. A função-lucro individual pode ser expressa matematicamente por:

$$
\mathrm{W}_{\mathrm{i}}\left(\mathrm{z}_{\mathrm{i}}, \mathrm{y}_{\mathrm{i}}\right)=\iint^{\mathrm{y}_{\mathrm{i}}} \mathrm{d}_{\mathrm{i}}\left(\eta_{\mathrm{i}}\right) \mathrm{d} \eta_{\mathrm{i}}-\int^{\mathrm{z}_{\mathrm{i}}} \mathrm{s}_{\mathrm{i}}\left(\varepsilon_{\mathrm{i}}\right) \mathrm{d} \varepsilon_{\mathrm{i}}
$$

O total ou função-lucro é:

$$
\mathrm{W}=\sum_{\mathrm{i}} \mathrm{W}_{\mathrm{i}}\left(\mathrm{z}_{\mathrm{i}}, \mathrm{y}_{\mathrm{i}}\right)
$$

Se $x_{i j}$ representa o total de movimentações aceitáveis de $i$ para $j$ com custo de transporte $t_{i j}$, então a expressão para lucro pode ser descrita como:

$$
\mathrm{NW}=\mathrm{W}-\sum_{\mathrm{i}} \sum_{\mathrm{J}} \mathrm{t}_{\mathrm{ij}} \mathrm{x}_{\mathrm{ij}}
$$

Para que o chamado equilíbrio espacial seja observado, requere-se que os fluxos direcionados à região $j$ sejam maiores ou iguais à demanda da região $j$, então:

$$
\mathrm{y}_{\mathrm{j}}<\sum_{\mathrm{i}} \mathrm{x}_{\mathrm{ij}} \text {, para todo } \mathrm{j}
$$

e que os fluxos originados na região $i$ não sejam maiores que as quantidades ofertadas pela região $i$. Então:

$$
\mathrm{z}_{\mathrm{i}} \geq \sum_{\mathrm{j}} \mathrm{x}_{\mathrm{ij}} \text {, para todo } \mathrm{i}
$$

Quando as funções de oferta e demanda são lineares, i.e.,

$$
\mathrm{p}_{\mathrm{i}}=\mathrm{a}_{\mathrm{i}}-\mathrm{b}_{\mathrm{i}} \mathrm{y}_{\mathrm{i}} \quad \text { e } \quad \mathrm{p}^{\mathrm{i}}=\mathrm{c}_{\mathrm{i}}-\mathrm{d}_{\mathrm{i}} \mathrm{z}_{\mathrm{i}}
$$

o problema será igual:

$$
\begin{aligned}
& \operatorname{Max}_{\sum_{\mathrm{i}}}\left(\mathrm{a}_{\mathrm{i}} \mathrm{y}_{\mathrm{i}}-1 / 2 \mathrm{~b}_{\mathrm{i}} \mathrm{y}_{\mathrm{i}}^{2}-\mathrm{c}_{\mathrm{i}} \mathrm{z}_{\mathrm{i}}-1 / 2 \mathrm{~d}_{\mathrm{i}} \mathrm{z}_{\mathrm{i}}^{2}\right)-\sum_{\mathrm{i}} \sum_{\mathrm{j}} \mathrm{t}_{\mathrm{ij}} \mathrm{x}_{\mathrm{ij}} \\
& \mathrm{y}_{\mathrm{j}}<\sum_{\mathrm{i}} \mathrm{x}_{\mathrm{ij}} \text {, para todo } \mathrm{j} \\
& \mathrm{z}_{\mathrm{i}} \geq \sum_{\mathrm{j}} \mathrm{x}_{\mathrm{ij}} \text {, para todo } \mathrm{i} \\
& \mathrm{y}_{\mathrm{j}}, \mathrm{z}_{\mathrm{i}}, \mathrm{x}_{\mathrm{ij}} \geq 0
\end{aligned}
$$

O termo quadrático incluído na função-objetivo (10) é a razão do grau de não-linearidade do modelo proposto. A solução sugerida pelo modelo determina o fluxo de soja-grão das regiões de oferta para as regiões de demanda doméstica, portos e demanda internacional.

No modelo, inicialmente, foram identificadas as regiões de oferta e demanda de soja. A escolha das regiões que compõem o modelo partiu de uma Análise de Mercado da Commodity Soja, considerando, também, a infra-estrutura disponível. Neste estudo, foram analisados o comportamento dos últimos anos das variáveis produção de soja, rendimento médio, área cultivada, exportações e capacidade instalada de processamento (planta industrial). A escolha dos Estados que compõe o modelo, partiu do intuito de tentar caracterizar o mercado, considerando as regiões que tiveram desenvolvimento mais predominante nos últimos anos e potencial de expansão com base nas fronteiras agrícolas em constante crescimento. 
Para a caracterização das regiões de excesso de oferta e demanda, partiu-se da seguinte premissa: se a produção de soja for maior que a quantidade processada, essa região fica caracterizada como região de excesso de oferta, caso contrário fica caracterizada como região de excesso de demanda. Para os Estados do Paraná, Mato Grosso e Mato Grosso do Sul, foram identificadas diferentes microrregiões, tanto com relação à produção como ao processamento.

O processamento das informações para o modelo de equilíbrio espacial desenvolvido para a movimentação de soja no Brasil foi feito utilizando-se do programa computacional General Algebraic Modeling System - GAMS (BROOKE et al., 1995), e a programação matemática adotada foi a não-linear (função-objetivo não-linear e restrições lineares). A sub-rotina (MINOS) utilizada para resolver o problema é uma junção do método do Gradiente reduzido (LUENBERGER, 1984) com o método quasi-Newton.

O objetivo do modelo de equilíbrio é maximizar a função lucro-total definida por SAMUELSON (1952) usando a estrutura básica proposta por TAKAYMA \& JUDGE (1971). Os dados que alimentaram o modelo (produção, consumo, preços de comercialização do mercado nacional e internacional, fretes dos diferentes modais) tiveram como base o ano de 2002. Os dados de produção tiveram como fonte o Instituto Brasileiro de Geografia e Estatística (IBGE) e o Departamento de Agricultura dos Estados Unidos (USDA). O consumo teve como base a Associação Brasileira de Indústrias de Óleos Vegetais (ABIOVE), e as cotações da soja para os mercados nacional e internacional tiveram como base a consultoria SAFRAS \& Mercado e o USDA, respectivamente. Já os dados de elasticidades-preço de oferta e demanda foram baseados nos estudos desenvolvidos por FULLER et al. (2003).

Os custos de transporte dos modais rodoviário e ferroviário no modelo foram estimados por meio de equações lineares baseadas nas distâncias entre os pontos de carregamento e os de recepção (origem/destino). O comportamento do custo dos modais (variável de resposta) foi analisado por meio de modelo de regressão linear múltipla, utilizando banco de dados de fretes praticados em 2002, com base nos dados do Sistema de Informação de Fretes (SIFRECA), em todo o território brasileiro, de acordo com a distância e os diferentes meses do ano. Para o modal hidroviário, foram utilizados os fretes praticados.

Desse modo, para o modal rodoviário, partiu-se da hipótese de existir comportamento diferenciado para fretes com distâncias de até $500 \mathrm{~km}$ e com sazonalidade de preços para o primeiro e segundo semestres do ano (variáveis explicativas).

Assim, para o modal rodoviário, tem-se:

$$
\begin{aligned}
& Y=X \beta+\varepsilon \\
& Y=\beta_{0}+\beta_{1}\left(1-\delta_{1}\right)\left(1-\delta_{2}\right) X+\beta_{2}\left(1-\delta_{1}\right) \delta_{2} X+\beta_{3} \delta_{1}\left(1-\delta_{2}\right) X+\beta_{4} \delta_{1} \delta_{2} X+\varepsilon \\
& \delta_{1}=\left\{\begin{array}{l}
0 \text { se distância }<500 \\
1 \text { se distância } \geq 500
\end{array} \quad \delta_{2}=\left\{\begin{array}{l}
0 \text { se semestre }=1 \\
1 \text { se semestre }=2
\end{array}\right.\right.
\end{aligned}
$$

em que,

$\mathrm{Y}$ - frete (variável dependente), $\mathrm{R} \$ \mathrm{t}^{-1}$;

$\mathrm{X}$ - distância (variável independente), km;

$\beta$ - coeficiente da regressão;

$\varepsilon$ - erro aleatório;

$\delta_{1}$ - distância (variáveldummy), e

$\delta_{2}$ - semestre (variáveldummy).

O modelo linear de custo foi implementado no programa estatístico Minitab 13.0. Na Figura 2, ilustra-se o comportamento dos custos rodoviários observados e estimados pela regressão. 


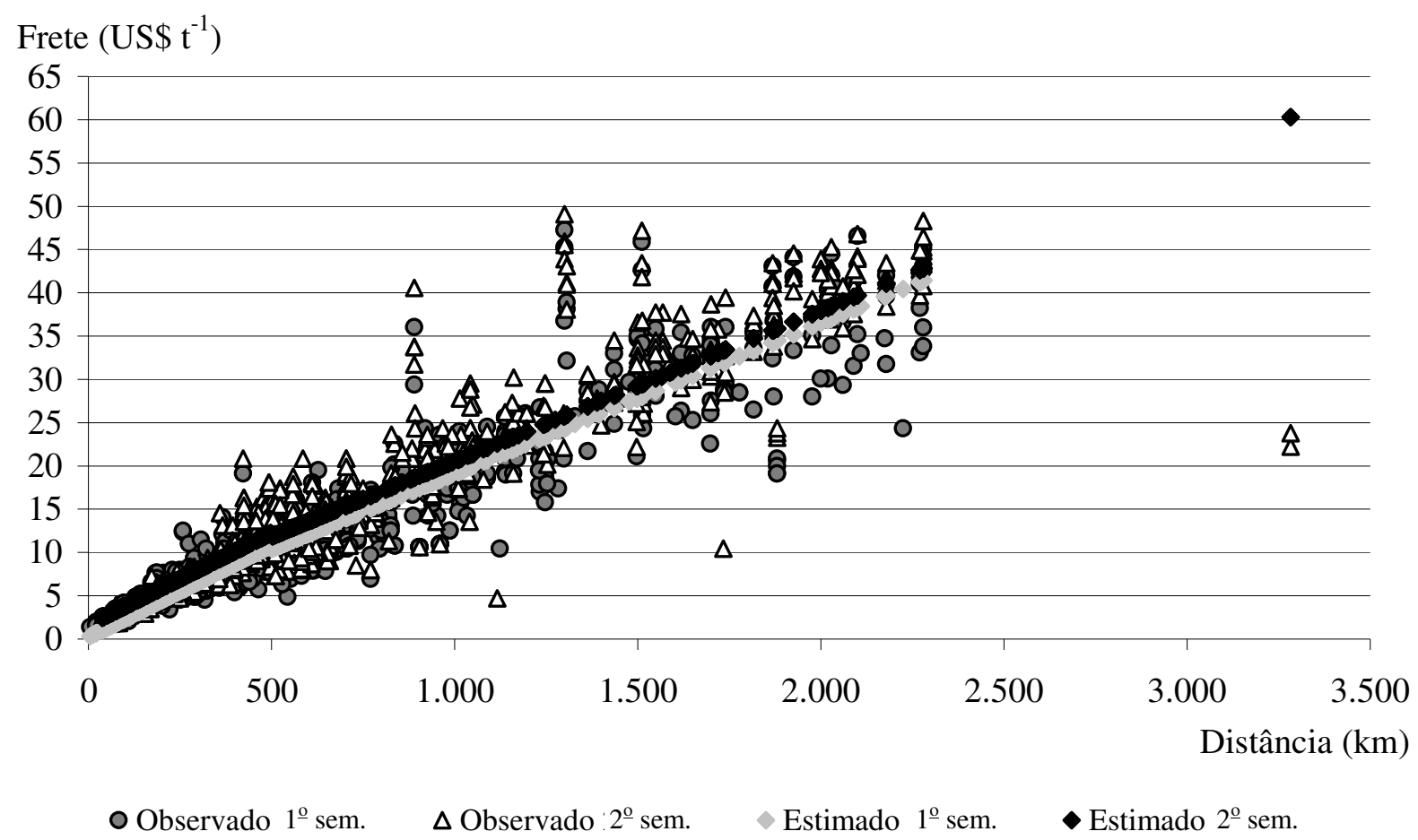

FIGURA 2. Dispersão dos custos rodoviários por quilômetro.

Para o transporte ferroviário, as equações foram desenvolvidas da mesma maneira que para o modal rodoviário, não incluindo a variável semestre, visto que, para essa variável explicativa, não se obteve significância estatística quando foi inserida no modelo e foi observado comportamento linear dos preços ferroviários durante o ano, sendo a distância-referência encontrada de $850 \mathrm{~km}$.

\section{RESULTADOS E DISCUSSÃO}

O modelo de equilíbrio espacial obtido para a movimentação da soja brasileira passou por um processo de verificação e validação dos dados para a determinação da sua confiabilidade e utilidade, podendo assim gerar cenários futuros. O primeiro passo desenvolvido foi a verificação dos dados resultantes, analisando-se os fluxos gerados entre a oferta e a demanda de soja nas respectivas regiões e nos dois semestres envolvidos, com ênfase nas quantidades comercializadas e preços de oferta. Na Tabela 2, pode-se verificar que as quantidades ofertadas e demandas pelo modelo foram equivalentes.

TABELA 2. Oferta e demanda de soja.

\begin{tabular}{lcc|lc}
\hline Oferta & Volume $($ mil t $)$ & Preço $\left(\mathrm{US} \$ \mathrm{t} \mathrm{t}^{-1}\right)$ & Demanda & Volume (mil t) \\
\hline BA & 158,4 & 159,0 & MS-CN & 241,7 \\
RO & 363,3 & 157,4 & MT-CS & 253,6 \\
MA & 573,8 & 169,8 & MT-SD & 292,8 \\
PR-CO & $1.514,8$ & 173,0 & PR-NC & 347,4 \\
MS-SO & $1.667,7$ & 161,8 & AM & 523,2 \\
PR-SO & $1.682,4$ & 172,4 & SP & $1.358,1$ \\
PR-O & $1.941,8$ & 172,4 & PR-SD & $1.473,8$ \\
MG & $2.720,4$ & 172,0 & Subtotal & $4.490,6$ \\
MT-NO & $3.637,1$ & 150,5 & Japão & $2.793,3$ \\
GO & $3.662,4$ & 162,7 & China & $8.914,3$ \\
RS & $3.951,6$ & 173,8 & Europa & $10.529,9$ \\
MT-N & $4.854,4$ & 150,0 & Subtotal & $22.237,5$ \\
\hline Total & $26.728,1$ & - & Total & $26.728,1$ \\
\hline
\end{tabular}

PR-SO: Sudoeste; PR-O: Oeste; PR-CO: Centro-Oeste; PR-SD: Sudeste; PR-NC: Centro-Norte; MT-N: Norte; MTNO: Noroeste; MT-SD: Sudeste; MT-CS: Centro-Sul; MS-SO: Sudoeste; MS-CN: Centro. 
Segundo FELLIN (1993), os procedimentos de validação de modelos podem variar e são necessários para interpretar e analisar o comportamento dos resultados do modelo. Assim, os dados estimados pelo modelo foram analisados por meio de estatísticas de correlação que indicaram alto grau de correlação entre as estimativas do modelo e os dados reais.

Na Tabela 3, apresenta-se a estimativa pontual para o coeficiente de correlação, para um intervalo de confiança de $95 \%$, e o valor-p do teste com a hipótese nula (que o coeficiente tenha valor zero).

Para a oferta, os dados foram confrontados com os dados do MDIC em 2002, enquanto os preços de equilíbrio, demanda doméstica e demanda internacional foram comparados com as cotações realizadas pelo Grupo SAFRAS \& Mercado, dados da ABIOVE e da USDA, respectivamente.

TABELA 3. Coeficiente de correlação entre as estimativas e os valores reais.

\begin{tabular}{lcc}
\hline Variável & Correlação & Valor-p \\
\hline Exportação & 0,916 & 0,0010 \\
Preço & 0,789 & 0,0020 \\
Demanda & 0,999 & 0,0000 \\
\hline
\end{tabular}

$\mathrm{Na}$ Tabela 4, apresentam-se os fluxos obtidos pelo modelo. O modal rodoviário foi vocacionado para o abastecimento interno e interligação dos modais que, em geral, ocorrem para distâncias inferiores a $850 \mathrm{~km}$.

Os modais ferroviário e hidroviário foram ativados para o transporte de longas distâncias com destino ao mercado internacional.

TABELA 4. Fluxo de movimentação de soja obtido pelo modelo.

\begin{tabular}{|c|c|c|c|c|}
\hline Origem & Destino 1 & \multicolumn{3}{|c|}{ Destino 2} \\
\hline Oferta & Demanda & Transbordo & Porto & Demanda Internacional \\
\hline $\begin{array}{c}\text { RO } \\
\text { MT-NO }\end{array}$ & $\mathrm{AM}(\mathrm{r})$ & P. Velho (h) & Itacoatiara $(\mathrm{h})$ & China e Japão (m) \\
\hline $\begin{array}{l}\text { BA } \\
\text { MA }\end{array}$ & & $\begin{array}{l}\text { Araguari (f) } \\
\text { Estreito (f) }\end{array}$ & $\begin{array}{l}\text { Vitória (f) } \\
\text { Itaqui (f) }\end{array}$ & China (m) \\
\hline MS-SO & $\begin{array}{c}\text { MS-CN (r) } \\
\text { SP (r) } \\
\text { PR-NC (r) }\end{array}$ & Londrina (f) & Paranaguá (f) & China (m) \\
\hline PR-O & & & Paranaguá (f) & China (m) \\
\hline PR-CO & PR-NC (f) & & Paranaguá (f) & China (m) \\
\hline PR-SO & PR-SD (f) & & Paranaguá (f) & China $(\mathrm{m})$ \\
\hline MT-N & $\begin{array}{l}\text { MT-SD (r) } \\
\text { MT-CS (r) }\end{array}$ & A. Araguaia (f) & Santos (f) & Europa (m) \\
\hline GO & $\begin{array}{l}\text { MT-SD (r) } \\
\text { SP (r) }\end{array}$ & Goiânia (f) & Santos (f) & Europa (m) \\
\hline MG & $\mathrm{SP}(\mathrm{r})$ & & Santos (f) & Europa (m) \\
\hline $\mathrm{RS}$ & & & Rio Grande (f) & Europa, China e Japão (m) \\
\hline
\end{tabular}


Um novo cenário (B) foi proposto, alterando-se, sensivelmente, os custos de transporte ferroviário e hidroviário em $20 \%$ e o rodoviário mantido. Os fluxos mantiveram-se com aumento nos níveis de volume transportados pelos modais ferroviário e hidroviário, 5,3\% e 3,2\%, respectivamente; em decorrência da redução dos custos, foi verificada também alteração nos preços de equilíbrio.

As políticas para o setor de transportes devem ser direcionadas para incentivar a intermodalidade, visto que os modais viários são interdependentes e cada um deles apresentando determinada vocação, tendo vantagens e desvantagens com relação as distâncias percorridas, ao tipo de carga, à quantidade transportada e ao tempo percorrido entre origem e destino.

Uma plena adequação do sistema não depende apenas de malha viária disponível e em condições de uso, mas também da estrutura complementar, como armazéns e terminais ferroviário, hidroviário e marítimo.

Por exemplo, atualmente, têm-se dois exemplos importantes nessa direção. O primeiro é a implantação da hidrovia Tocantins-Araguaia, planejada para o transporte da região Centro-Oeste para o Porto de Belém (PA) para exportação, ou ligando a região até o terminal hidroviário Porto Franco (MA), seguindo por ferrovia até o Porto de Itaqui (MA) para exportação. O segundo seria o prolongamento da Ferronorte, ferrovia que interliga o Estado do Mato Grosso ao Porto de Santos, até o município de Rondonópolis (MT). Ambos são bons exemplos do esforço que o governo federal tem feito para o setor de transportes brasileiro. Tais investimentos estão em fase embrionária, mas apontam para uma política preocupada em fomentar condição mais favorável.

\section{CONCLUSÕES}

O desenvolvimento do modelo de equilíbrio espacial e os resultados obtidos para a logística de movimentação da soja brasileira foram satisfatórios. Dessa forma, pode-se chegar a resultados que permitem inferir cenários futuros para análise da competitividade da soja brasileira, por meio das melhorias dos sistemas de transportes associados a novos projetos viários.

As técnicas estatísticas de correlação empregadas para a validação do modelo foram significativas para as variáveis analisadas (oferta doméstica, demanda doméstica, demanda internacional e variável preço de oferta doméstica).

Um novo cenário (B) foi proposto, no qual os custos de transporte ferroviário e hidroviário foram reduzidos em $20 \%$ e o rodoviário mantido. Com essa alteração, verificou-se aumento nos níveis de volume transportados pelos modais ferroviário e hidroviário como conseqüência da redução dos custos, e ainda sensível alteração nos preços de equilíbrio. Assim, redistribuir de maneira eficiente o transporte de carga no Brasil pode potencializar a sua inserção no mercado internacional.

Dessa forma, existe a possibilidade de utilização do modelo para testar diferentes hipóteses, considerando as melhorias nos sistemas de transporte que podem acarretar em redução dos custos com frete envolvidos, ou seja, em trabalhos futuros, pode-se incluir cenário hipotético, como nova rota de escoamento da produção com a viabilização de trecho hidroviário ou ferroviário, verificando-se o seu impacto no contexto geral.

A estrutura teórica desse modelo pode ser expandida, incluindo regiões multiexportadoras e importadoras e multicommodity. A introdução de novos dados ao modelo, incluindo mais regiões de excesso de oferta e demanda, portos, demanda internacional e novos fluxos de transporte poderão gerar resultados que mais se aproximem dos valores ótimos que possam garantir maior inserção do Brasil no mercado internacional da commodity soja.

\section{REFERÊNCIAS}

BROOKE, A.; KENDRICK, D.; MEERRAUS, A. GAMS: a user's guide. Release 2.25. Redwood: The Scientific Press, 1995. 289 p. 
CAIXETA FILHO, J.V. Transporte e logística no sistema agroindustrial. Preços Agrícolas: mercados agropecuários e agribusiness, Piracicaba, v.10, n.119, p.2-7, set. 1996.

CONFEDERAÇÃO NACIONAL DO TRANSPORTE. Boletim Estatístico - CNT. dezembro 2005. Seção Boletim Estatístico. Disponível em:

$<$ http://www.cnt.org.br/cnt/downloads/becnt/becnt_122005.pdf>. Acesso em: 20 dez. 2005.

FELLIN, L.R. International corn and soybean transportation system: quadratic programming models. 1993. 134 f. Tese (Doutorado em Economia Agrícola) - Texas A\&M University, College Station, 1993.

FULLER, S.; YU, T.; FELLIN, L.; LALOR, A.; KRAJEWSKI, R. Transportation developments in south america and their effect on international agricultural competitiveness. Transportation Research Board - Journal of the Transportation Research Board, Washington, n.1820, p.62-8, 2003.

LíCIO, A. Os eixos estruturadores e dos corredores de transportes. Revista de Política Agrícola, Brasília, v.9, n.4, p.3-4, 1995.

LIMA, L.M.; BRANCO, J.E.H.; CAIXETA FILHO, J.V. Um modelo dinâmico para otimização do escoamento de soja em grão. In: CONGRESSO DA SOCIEDADE BRASILEIRA DE ECONOMIA E SOCIOLOGIA RURAL, 43., 2005, Ribeirão Preto. Anais... Ribeirão Preto: Sociedade Brasileira de Economia e Sociologia Rural, 2005. 17 p.

LUENBERGER, D.G. Linear and nonlinear programming. $2^{\text {nd }}$ ed. Addison: Wesley Reading, 1984. $491 \mathrm{p}$.

OJIMA, A.L.R.O. Análise da movimentação logística e competitividade da soja brasileira: uma aplicação de um modelo de equilíbrio espacial de programação quadrática. 2004. 79 f. Dissertação (Mestrado Automação) - Faculdade de Engenharia Elétrica e de Computação, Universidade Estadual de Campinas, Campinas, 2004.

SAMUELSON, P.A. Spatial price equilibrium and linear program. American Economic Review, Stanford, v.42, p.283-303, 1952.

TAVARES, C.E.C. Fatores Críticos à competitividade da soja no Paraná e no Mato Grosso. Brasília, julho, 2004. Disponível em:

$<$ http://www.conab.gov.br/download/cas/especiais/Trabalho\%20sobre\%20Competitividade\%20Soj a\%20MT\%20e\%20PR.pdf>. Acesso em: 20 dez. 2005.

TAKAYAMA, T.; JUDGE, G.G. Spatial and temporal price and allocation models. Amsterdan: North Holland Publishing, 1971. 528 p. 